\title{
Percolation of Phase-Separating Polymer Mixtures
}

\author{
Di-Yao Hsu, Che-Min Chou, ${ }^{\dagger}$ Ching-Yen Chuang, and Po-Da Hong* \\ Department of Materials Science and Engineering, National Taiwan University of Science \\ and Technology, Taipei, 10607, Taiwan \\ †chemin.chou@mail.ntust.edu.tw \\ *poda@mail.ntust.edu.tw
}

\section{SI-1. Sample Purification}

The poly(ethylene glycol) (PEG) [Lot Num. BCBF9503V, $\bar{M}_{w}=23,245$ and $\bar{M}_{w} / \bar{M}_{n}=1.25(\mathrm{COA}$ of the product), Sigma-Aldrich Co.] and the poly(ethylene glycol-ran-propylene glycol) random copolymer (RAN) [Lot Num. MKBG3977V, ethylene glycol content of about $73.3 \%, \bar{M}_{n} \sim 12,000$, (COA of the product), Sigma-Aldrich Co.] were used in this work. The samples have to be purified for use in the experiments. For PEG flakes, the $10 \mathrm{wt} \%$ solution was prepared by dissolved the polymer flakes into the deionized water with stirring at the room temperature. Then, the solution goes through two stages of filtration [0.45 $\mu \mathrm{m}$ PVDF filter (Durapore ${ }^{\circledR}$ Membrane filter) and $0.22 \mu \mathrm{m}$ PVDF filter (Millipore Millex ${ }^{\circledR} \mathrm{GV}$ filter)]. The clean solution was stirred on a hot plate at $353 \mathrm{~K}$ until all the excess water was evaporated. Finally, the dried PEG sample was obtained by vacuum drying at room temperature for 3 days. For RAN viscous liquid, we apply ultracentrifuge (Beckman Coulter, Optima L-90K with type 90 Ti rotor) method to remove the impurities. The sample was sealed in the OptiSeal ${ }^{\mathrm{TM}}$ polyallomer tube $(8.9 \mathrm{~mL})$ and centrifuged at $80,000 \mathrm{rpm}$ for 2 hours at 308 K. Carefully decant approximately $50 \%$ of the supernatant liquid into the clean bottle.

\section{SI-2. Turbidimetry}

Since the conventional turbidity measurement involves kinetic effects, the differential refractometry is a better way in terms of an accurate determination of the coexistence curve. ${ }^{1-4}$ However, it would be difficult to undertake experiments for highly viscous polymer mixtures. In our turbidity measurement, we were able to avoid this dilemma by using a more delicate experimental protocol to determine the coexistence curve of the PEG/RAN mixtures.

The experimental setup of our turbidimetry is sketched in Figure S1. The light source was the fiber-coupled laser system ( $5 \mathrm{~mW}$ polarized He-Ne laser with an $11 \mathrm{~mm}$ EFL aspheric collimator). 


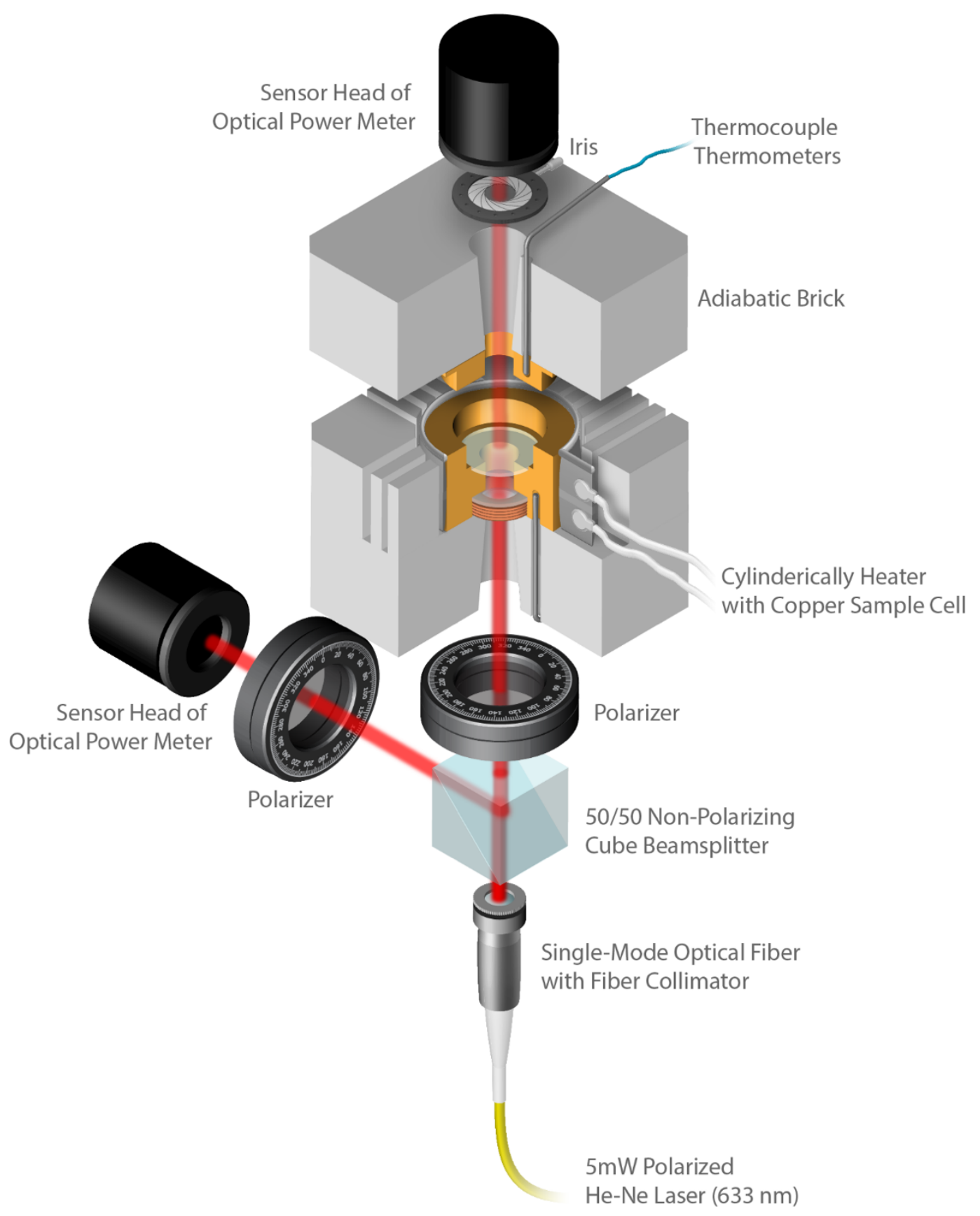

Figure S1. Schematic diagram of turbidimetry apparatus.

The collimated light beam was passed through the non-polarizing 50:50 beamsplitter cube and shined onto the sample cell and the reference beam detector, respectively. The polarizers placed in front of the sample and detector were used to modulate the analyzed direction for balancing the initial light intensities. The copper sample cell is tubular, of path length $L=10 \mathrm{~mm}$ and internal diameter of $10 \mathrm{~mm}$, and is sealed by two quartz cover slips, of $0.5 \mathrm{~mm}$ thickness. The band heater (Watlow ${ }^{\circledR} \mathrm{STB}$ 1J1A1) was wrapped the cell and was controlled by the temperature controller (RCK Instrument Inc., 
REX-P200). The iris placed in front of the transmitted beam detector was used to filter the scattered light from the sample.

In the experiment, the homogeneous polymer mixtures were first heated to $433 \mathrm{~K}$ for 20 min to stabilize the samples, and then cooled to $293 \mathrm{~K}$ with a cooling rate of $0.5 \mathrm{~K} / \mathrm{min}$ to monitor the intensity of the transmitted light. The turbidity is defined by ${ }^{5}$

$$
\tau=-\frac{1}{L} \ln \frac{I}{I_{0}}
$$

where $L$ is the path length in the cell, $I_{0}$ is the intensity of the reference light, and $I$ is the intensity of the transmitted light.

\section{SI-3. Other Measurements}

Gel Permeation Chromatography (GPC) $-\bar{M}_{w}$ and $\bar{M}_{w} / \bar{M}_{n}$ of the purified samples were determined by GPC [Waters ${ }^{\circledR} 515$ HPLC Pump and Waters ${ }^{\circledR} 2410$ Refraction Index (RI) Detector]. For the calibration, the polystyrene standards $\left[\bar{M}_{w}\left(\bar{M}_{w} / \bar{M}_{n}<1.10\right): 387,000,43,700,18,100\right.$, 6,520, and 2,950] were used and dissolved in the Tetrahydrofuran (THF) (LC grade) at $308 \mathrm{~K}$. The two columns [Waters ${ }^{\circledR}$ WATO-44237(HR2) and Waters ${ }^{\circledR}$ WATO-44225(HR4)] were used with a flow rate of $1 \mathrm{~mL} / \mathrm{min}$. For the measurement, the $0.005 \mathrm{~g} / \mathrm{ml} \mathrm{PEG/THF} \mathrm{and} \mathrm{RNA/THF} \mathrm{were} \mathrm{prepared,}$ respectively. The measurement condition was the same as the calibration, and the Waters ${ }^{\circledR}$ WATO44225(HR4) was used.

Small-Angle Light Scattering (SALS) — For the measurement, the quartz sample cell was placed on the temperature controlled stage (Linkam Scientific Co., THMS-600), and the scattering patterns were captured by a 16-bit back-illuminated CCD camera (Apogee Imaging Systems, Inc., Alta F47). Morphological Identification - The specimens were sealed between two quartz coverslips with a $10 \mu \mathrm{m}$ spacer and observed by phase contrast microscopy (Leica DMLP with Leica DFC490 CCD camera).

Rheological Measurement - The zero shear viscosity of the coexisting phases was measured by Anton Paar ${ }^{\circledR}$ MCR302 Rheometer with a $25 \mathrm{~mm}$ cone-plate geometry ( 0.017 rad with the gap 0.05 $\mathrm{mm})$. The temperature was controlled by the P-ETD 400 lower plate system and the H-ETD 400 hood. The shear rate range of the measurement is from $10^{-2}-10 \mathrm{~s}^{-1}$.

\section{SI-4. Parameter Estimation}

Since the mean-field nature has been experimentally confirmed, the polymer-polymer interaction parameter $\chi$ can easily be evaluated from the Flory-Huggins theory ${ }^{6}$ 


$$
\chi=\frac{v_{0}}{2 \Delta \phi}\left[\frac{1}{v_{1} N_{1}} \ln \left(\frac{\phi^{\prime \prime}}{\phi^{\prime}}\right)+\frac{1}{v_{2} N_{2}} \ln \left(\frac{1-\phi^{\prime}}{1-\phi^{\prime \prime}}\right)\right]
$$

where $v$ is the molar volume and $N$ is the the degree of polymerization; the subscript 0 denotes an arbitrary reference volume; the subscript 1 and 2 denotes the PEG and the RAN, respectively. Here, $v_{1}=38.9 \mathrm{~cm}^{3} \mathrm{~mol}^{-1}, v_{2}=44 \mathrm{~cm}^{3} \mathrm{~mol}^{-1}$ (calculated by an EG:PG $=3: 1$ and the molar volume of PPG is $58.1 \mathrm{~cm}^{3} \mathrm{~mol}^{-1}$ ), and $v_{0}=41.45 \mathrm{~cm}^{3} \mathrm{~mol}^{-1}$ (the average of $v_{1}$ and $v_{2}$ ).

Given the uncertainty in $N$ values, an appropriate iterative procedure is needed. According to Eq. (1), the parameters $\chi(T), N_{1}$, and $N_{2}$ can be estimated under the constraint of $T_{c}=403.56 \mathrm{~K}$ and $\phi_{c}=0.458$. The procedure is as follows: (1) using reference inputs $N_{1}^{\mathrm{GPC}}$ and $N_{2}^{\mathrm{GPC}}$ to calculate $\chi(T)$; (2) obtained the coefficients of $\chi(T)$ via the linear least-squares fitting; (3) solving $N_{1}^{\text {calc }}$ and $N_{2}^{\text {calc }}$ from the constraint of $\chi_{c}$ (i.e., $\chi$ at $T_{c}$ ) and $\phi_{c}$; (4) using $N_{1}^{\text {calc }}$ and $N_{2}^{\text {calc }}$ as new reference inputs and repeatedly optimizing $\chi(T), N_{1}$, and $N_{2}$. In fact, in the present work, $N_{1}$ and $N_{2}$ were quickly converged at two iterations. We obtained $N_{1}=422, N_{2}=267$, and $\chi(T)=-0.0103+$ $6.583 / T$.

On the other hand, the melting curve of the PEG is calculated by the Nishi-Wang equation ${ }^{8}$

$$
\frac{1}{T_{m}}-\frac{1}{T_{m}^{0}}=-\frac{R v_{1}}{\Delta H v_{2}}\left[\frac{\ln \phi}{N_{1}}+\left(\frac{1}{N_{1}}-\frac{1}{N_{2}}\right)(1-\phi)+\chi(1-\phi)^{2}\right]
$$

where in addition to above parameters, the melting temperature $T_{m}^{0}$ and the enthalpy of fusion $\Delta H$ are $336 \mathrm{~K}$ and 1,623 $\mathrm{cal} \mathrm{mol}^{-1}$ (calorimetry, DSC), respectively.

\section{Reference}

(1) Nakata, M.; Kuwahara, N.; Kaneko, M. J. Chem. Phys. 1974, 62, 4278-4283.

(2) Nose, T. Polymer 1995, 36, 2243-2248.

(3) Kuwahara, N.; Sato, H.; Kubota, K. Phys. Rev. Lett. 1995, 75, 1534-1537.

(4) Xia, K. Q.; An, X. Q.; Shen, W. G. J. Chem. Phys. 1996, 105, 6018-6025.

(5) Puglielli, V. G.; Ford, N. C. Phys. Rev. Lett. 1970, 25, 143-147.

(6) Flory, P. J. Principles of Polymer Chemistry; Cornell University Press: New York, 1953.

(7) Van Krevelen, D. W.; Nijenhuis, te, K. Properties of Polymers, 4 ed.; Elsevier: New York, 2009.

(8) Nishi, T.; Wang, T. T. Macromolecules 1975, 8, 909-915. 